\title{
POLYPHENOLS WITH ALGICIDAL ACTIVITY IN THE SUBMERGED MACROPHYTE MYRIOPHYLLUM SPICATUM L.
}

Elisabeth M. Gross \& Rainer Sütfeld

MPI Limnology, Dept. Ecophysiology, August-Thienemann-Str. 2, D-24306 Plön, FRG

Keywords: Myriophyllum spicatum, submerged macrophyte, gallic acid, ellagic acid, polyphenol, chemical defence, allelopathy.

\begin{abstract}
The role of phenols as defence mechanisms in various terrestrial plants is well studied. However, in aquatic environments, they are probably involved also in the chemical interactions between species. A periodic shift from dominance by submerged macrophytes to dominance by phytoplankton and vice versa without apparent change in the external nutrient loading is sometimes observed in shallow eutrophic lakes. Due to the fact that nutrient levels are not usually limiting to phytoplankton growth, the release of allelochemicals by macrophytes is suspected to be responsible for poor algal growth.

Myriophyllum spicarum is known to produce several phenolic compounds. Aqueous methanolic extracts of fresh or lyophilized as well as axenic or non-axenic $M$. spicatum plant material exhibited strong algicidal activity against cyanobacteria. Chlorophytes and diatoms are also affected, but to a lesser extent. Bioassay directed fractionation of the extract lead to the isolation of two strong algicidal compounds. These compounds are constitutively present in the plant and were characterized as galloyl glycosides (500 - 1000 dalton). To test the ecological importance of these substances, the culture water was screened for exudated phenolic compounds. At least 5 - 10 different polyphenols could be detected. An algicidal galloyl ester and several derivatives of ellagic acid were among them. The possible release mechanisms and the mode of action of these polyphenols as defence compounds are discussed.
\end{abstract}

\section{Introduction}

Recent studies (Scheffer et al., 1993) show that shallow eutrophic lakes can have two altemative stable equilibria: a clear state dominated by macrophytes and a turbid state dominated by high algal biomass. Both equilibria are possible over a wide range of nutrient levels. As both states are selfreinforcing, a certain degree of disturbance of the system is necessary to change from one state to the other. Many factors are involved. Nutrient levels, water depth and resuspension of sediment by waves or fish are especially important. The release of allelopathic substances (Wium-Anderson, 1987) is hypothesized to be a self-stabilizing mechanism of the clear state with macrophytes. By this, submerged macrophytes may compete well with algae for light and nutrients.

Several reports on the inhibitory activity of chemicals from Myriophyllum spicatum have been published. Planas et al. (1982) demonstrated the presence of at least 18 different phenolic compounds by TLC. The major compounds were identified as ellagic, gallic and sinapic acid. Low concentrations of the phenolic extract prolonged the growth lag time of Anacystis (cyanobacteria) and Selenastrum (chlorophyceae), whereas higher concentrations inhibited growth completely. Recently, several hydrolyzable polyphenols with algicidal activity have been isolated from other Myriophyllum species (Saito et al., 1989; Aliotta et al., 1992). All of these polyphenols exhibit a rather low molecular weight (ranging from approximately 500 to 1000 dalton) and contain at least one gallic acid residue. However, it is not known whether these substances are released into the environment in sufficient quantities to have adverse effects on algae. 
$M$. spicatum (Haloragacea) is a submerged macrophyte which grows at a depth of $1-5 \mathrm{~m}$ of the littoral zone of meso- to eutrophic lakes. It is able to built up dense stands and often forms canopies (Grace \& Wetzel, 1978). In the course of a screening for algicidal compounds from submerged macrophytes, we found that $M$. spicatum showed high inhibitory activity.

The purpose of this study was to evaluate whether hydrolyzable polyphenols from $M$. spicatum are responsible for the algicidal activity and to characterize these compounds. Further, the release of polyphenols into the surrounding water and their inhibitory action against algae was investigated.

\section{Material and methods}

\subsection{Plant material and cultivation}

Fresh plant material was collected from Schöhsee, a mesotrophic lake in northern Germany. Shoots were rinsed with tap water to remove attached substrata. They were either cultivated at $7^{\circ} \mathrm{C}$ in lake water for a year round supply with fresh shoots or frozen with liquid nitrogen, mortared and lyophilized. Lyophilized material was stored at $-21^{\circ} \mathrm{C}$. Axenic plant material was obtained by surface sterilization with sodium hypochlorid and further cultivated following the procedures of Kane \& Gilman (1991). For investigations of the released compounds, a modified medium containing only mineral nutrients plus thiamine- $\mathrm{HCl}$ was used.

\subsection{Isolation of the algicidal polyphenols}

Fresh or lyophilized plant material was extracted twice using $\mathrm{MeOH}: \mathrm{H}_{2} \mathrm{O} 1: 1(\mathrm{v} / \mathrm{v})$ for $30 \mathrm{~min}$ in an ultrasonic bath. The combined extracts were filtered (Whatman GF/F). After evaporation of methanol, the water extract was sequentially partitioned with $\mathrm{Et}_{2} \mathrm{O}$. EtOAc and $\mathrm{l}-\mathrm{BuOH}$. After evaporation of the solvents in vacuo, the residues were redissolved in a small amount of EtOH. The EtOAc-fraction containing most of the inhibitory activity was further separated with RP-HPLC.

\subsection{HPLC}

Extracts or MeOH-eluates from SPE (solid phase extraction) were separated on a $250 * 4.6 \mathrm{~mm}$ C18-column (Kromasil, $5 \mu \mathrm{m}$, EKA Nobel). Samples were eluted with a gradient of increasing $\mathrm{MeOH}$ concentration in $\mathrm{H}_{2} \mathrm{O}$ containing $10 \mathrm{mM} \mathrm{KH}_{2} \mathrm{PO}_{4}$ and $10 \mathrm{mM} \mathrm{H}_{3} \mathrm{PO}_{4}$. A flow rate of $1 \mathrm{ml} / \mathrm{min}$ and a solvent gradient profile of (a) $30-60 \% \mathrm{MeOH}(0-20 \mathrm{~min})$, (b) $60-100 \% \mathrm{MeOH}(20-25 \mathrm{~min})$ and (c) $100 \% \mathrm{MeOH}(25-40 \mathrm{~min})$ was used.

\subsection{Bioassays}

The agar diffusion assay (ADA) was performed as previously described (Gross et al., 1991) with cyanobacteria and chlorophytes as test organisms. Usually, Anabaena variabilis, strain $\mathrm{P} 9$ was used. Due to the low contrast diatoms gave with the ADA, the following radiocarbon primary productivity bioassay was developed according to Steemann Nielsen (1952): $50 \mu \mathrm{l}$ extract or fractions were added to $4.6 \mathrm{ml}$ medium containing diatoms at an optical density at $530 \mathrm{~nm}$ of $20-100 \mathrm{mAU}$ (ca. $0.2-0.8 \mu \mathrm{g}$ $\mathrm{Chl} / \mathrm{ml}$ ). $500 \mu \mathrm{l} \mathrm{NaH}{ }^{14} \mathrm{CO}_{3}$ (ca. $0.2-0.5 \mu \mathrm{Ci}$ ) were added to start the incorporation of ${ }^{14} \mathrm{C}$. The reaction was stopped after $4 \mathrm{~h}$ with $250 \mu \mathrm{l}$ formole. The suspension was filtered on $0.2 \mu \mathrm{m}$ cellulose nitrate filters (BA83, Schleicher \& Schüll, FRG). To minimize mechanical damage of cells, a pressure of 0.2 bar was not allowed to exceed during filtration. Each filter was washed two times wit $2.5 \mathrm{ml}$ millipore water. The filters were' placed in scintillation vials containing $5 \mathrm{ml}$ Instant Scint plus $5 \%$ Carbosorb (Packard Inc.) and counted. Triplicates of each treatment were performed. Radioactivity was determined by liquid scintillation (1900CA Tri-Carb Liquid Scintillation Analyzer, Packard, Inc.). Inhibition rates were expressed in \% values of the controls.

\subsection{Polyvinylpyrrolidone (PVP) assay}

Per $\mathrm{ml}$ of extract $1 \mathrm{ml}$ PVP-suspension $(0.1 \mathrm{~g} \mathrm{PVP} / \mathrm{ml}$ millipore water) was added, vortexed and incubated overnight at $4^{\circ} \mathrm{C}$. The suspension was centrifuged $(4000 \mathrm{~g})$ for $5 \mathrm{~min}$ and the supernatant tested for phenolics or algicidal activities. 


\subsection{Folin assay}

The Folin assay was performed using the Folin-Ciocalteu reagent according to Box (1983). Gallic and tannic acid turned out to be suitable as calibration standards to express the phenol content of the culture water as "phenolic acid equivalents".

\subsection{SPE (solid phase extraction)}

Filtered (Whatman GF/F) culture water of $M$. spicatum (up to $500 \mathrm{ml}$ ) was passed over a preconditioned $500 \mathrm{mg}$ sorbent RP-C18-cartridge (ICT). The cartridge was washed with millipore water and then the compounds which had bound to the cartridge were desorbed with methanol. With this method, medium to low polar substanced can be trapped. SPE was also used to desalt fractions from HPLC.

\subsection{Ultrafiltration}

Ultrafiltration was performed with YCOS and YM1 membranes in a model 8200 ultrafiltration cell (Amicon) under $\mathrm{N}_{2}$ atmosphere.

\subsection{Enyzmatic hydrolysis with tannase}

HPLC-fractions containing the inhibitors were evaporated of $\mathrm{MeOH}$. The residual water phase was adjusted to $\mathrm{pH} 7.0$ by adding $10 \mathrm{mM} \mathrm{K}_{2} \mathrm{HPO}_{4}$ and $20 \mu \mathrm{l}$ of tannase dissolved in $10 \mathrm{mM}$ potassiumphosphatebuffer, $\mathrm{pH} 7.0$ ( $200 \mu \mathrm{g}$ of enzyme). For total hydrolysis, samples were kept overnight at room temperature. The reaction was stopped by adding TFA to a final concentration of $0.1 \%$. The mixture was tested for phenolic acids by HPLC and for glucose by TLC.

\subsection{TLC for glucose}

TLC for glucose was performed on cellulose plates (Merck) with EtOAC:acetic acid: $\mathrm{H}_{2} \mathrm{O}$ 3:1:1 $(\mathrm{v} / \mathrm{v} / \mathrm{v})$. Spots were detected with the anilin-phtalic acid reagent. Glucose was identified by reference material.

\section{Results}

\subsection{Isolation of algicidal polyphenols from $M$. spicatum}

Extracts from either fresh or lyophilizated $\boldsymbol{M}$. spicatum shoots exhibited strong inhibition against cyanobacteria. Water, methanol or mixtures of both solvents could be used to extract the biologically active compounds from the plant material. Finally, the extraction procedure was performed with $\mathrm{MeOH}: \mathrm{H}_{2} \mathrm{O}$ 1:1 ( $(\mathrm{v} / \mathrm{v})$. An aliquot of the crude extract equivalent to $10 \mathrm{mg}$ DW caused a clearing zone of $4 \mathrm{~cm}$ in diameter with Anabaena variabilis strain P9. The inhibition of chlorophytes (Scenedesmus, Nannochloris) was weaker by a factor of 10 - 20 measured with the ADA. The same amount of extract caused an $50-70 \%$ inhibition of the primary productivity of the diatoms Nitzschia palea and Gomponema parvulum in the radiocarbon assay compared to controls (ethanol as solvent). The same degree of inhibition measured as diameter of clearing zone in the ADA was found with extracts from both axenic and non-axenic plant material. Treatment of the extract of $\boldsymbol{M}$. spicatum with PVP resulted in a disappearance in inhibitory activity, indicating that phenolic compounds are responsible for the algicidal activity. RP-HPLC separation of the EtOAc fraction revealed at least two main inhibitory fractions determined with the ADA (see p. , Fig. 1a). These main inhibitors were named " $\mathrm{FX1"}$ " and "FX2". Subsequent enzymatic hydrolysis of these fractions with tannase lead to gallic acid, determined by HPLC (see p. , Fig. lb), and glucose, identified by TLC. Thus, at least two different galloyl glycosides seemed to be responsible for the algicidal activity. Ultrafiltration with 500 or 1000 cut-off membranes provided estimates of about 500 dalton for the molecular weight of FX1 and a range between 500 and 1000 dalton for FX2. Calculating the amount of released gallic acid after enzymatic hydrolysis and comparing these data to the minimal inhibitory concentration for gallic acid in the ADA showed, that the galloyl esters have around $10-100$ fold more inhibitory effect than the simple phenolic gallic acid. 


\subsection{Release of polyphenols into the culture water by $M$. spicatum}

RP-HPLC separation of SPE-enriched culture water revealed at least $5-10$ different UVabsorbing signals when compared with the culture medium as control (see p. , Fig 2). They disappeared, in part, after treatment of the eluate with PVP, thus indicating their phenolic origin. At Rt 6.3 min FX2 can be identified by retention time and UV-scan in comparision with FX2 obtained from the extract of shoots. At Rt 17.4 min ellagic acid was present, identified with the reference substance. The signals at Rt 13.1, 18.5 and $21.5 \mathrm{~min}$ all exhibited a higher absorbance at $254 \mathrm{~nm}$ than at $280 \mathrm{~nm}$. Comparing their UV-scan with that of ellagic acid suggested that these compounds are ellagic acid derivatives.

In order to study the accumulation of these polyphenols, the culture water was changed at increasingly longer time intervals, then tested with the Folin assay for total phenolics and with SPE and HPLC for single compounds. A steady increase in the total released phenolics was obvious from the Folin assay (see p. , Fig 3a), but single compounds exhibited variable patterns. For example, the release of FX2 seemed to increase first and then decreased at the end. The compound at Rt. 21.5 min, probably an ellagic acid derivative, showed a clear increase over time whereas another ellagic acid derivative at Rt 18.5 min showed no clear pattern (see p. , Fig 3b).

SPE-enriched culture water of $M$. spicatum inhibited cyanobacteria in the ADA. An aliquot equivalent to $250 \mathrm{ml}$ of culture water of a dense culture (ca. $1 \mathrm{~g} \mathrm{DW}$ ) exhibited a clearing area of 2 $\mathrm{cm}$ in diameter with $A$. variabilis $\mathrm{P} 9$. It seems likely, that at least some of the released polyphenols were responsible for this.

\section{Discussion}

This study shows that $M$. spicalum produces polyphenols that have a distinct algicidal activity. They are constitutively in the plant as the results with axenic plant material proved, thus no induction of the biosynthesis is caused by other organisms. Two different galloyl glycosides of low molecular weight are responsible for most of the algicidal activity. These results are in a good agreement with the studies of Saito et al. (1989) and Aliotta et al. (1992), who identified hydrolyzable polyphenols from other Myriophyllum species. All of these identified algicidal compounds have a molecular weight lower than 1000 dalton and they all contain at least one gallic acid residue. Thus, the production of such polyphenols seems to be a common feature in the genus Myriophyllum.

It was shown that $M$. spicatum releases several polyphenols into the surrounding water. One of the algicidal galloyl esters and several ellagic acid derivatives are present among them. Multiple release mechanisms have been reported from $M$. spicatum. First of all, especially young plant tissues have secretory trichomes. These trichomes are known to be responsible for a certain part of EOC (extracellular organic carbon) release (Nalewajko \& Godmaire, 1993) and they are known to contain "tannins" (Janson, 1918). Further, $M$. spicatum shows easy fragnentation. Vegetative dispersal by fragmentation is common for this macrophyte to spread (Grace \& Wetzel, 1978). As growth and senescence are coincidental processes in submerged macrophyte communities, a steady exudation of organic material by intact tissues as well as leaching of organic material by senescing shoots takes place.

Gallic and ellagic acid have been shown to inhibit alkaline phosphatase (Wetzel, 1992), which is an enzyme many algae need to make phosphorus available to them. More complex polyphenols exhibit a stronger binding to proteins (Haslam, 1989). This may explain the higher inhibitory activity of the isolated galloyl esters, compared with pure gallic acid. Further, polyphenols may bind ferrous ions. Again the binding is stronger in the high molecular weight polyphenols than in simple phenolics (Scalber, this volume). Iron becomes sometimes limiting, especially in hard water lakes. The release of polyphenols which are able to chelate ferrous ions may help the macrophyte to hold available iron. Macrophyte derived polyphenols contribute to the humic complex found in freshwater lakes. A steady complexation of the various polyphenolic compounds takes place as well as a continuous break down caused by photolysis or bacterial metabolism. Therefore, detection of polyphenols in situ may be very difficult because of the mentioned interactions with other humic compounds. However, the relcase of polyphenols from axenic cultures of $M$. spicatum under controlled environmental conditions can be 
qualitatively and quantitatively investigated. Coculture of $\boldsymbol{M}$. spicatum with algae or bacteria could deliver further information about possible interactions among species.

SPE enriched polyphenols from the culture water of $M$. spicatum inhibit cyanobacteria in the ADA. Further investigations will focus on the mechanism of this inhibition. It is likely that extracellular enzymes from the algae are affected by polyphenols released by $M$. spicatum. A target enzyme could be alkaline phosphatase.

In conclusion it can be stated that, apart from their role as herbivore deterrents, macrophyte derived polyphenols show algicidal activity against epiphytic (diatoms) and phytoplanktonic (cyanobacteria, chlorophytes) algae. From an ecological and evolutionary point of view, the production of allelochemicals against epiphytes seems to be more advantageous for submerged macrophytes because no dilution of the released inhibitors occurs. Until now, we do not know if the release of polyphenols by $M$. spicatum is an active process triggered by competing organisms or if it is a steady exudation or leaching of organic material. Appropriate bioassays are needed to investigate the release patterns of polyphenols under different environmental conditions. Further studies are in progress to investigate their role as self-stabilizing feature of the clear water state dominated by submerged macrophytes in shallow eutrophic lakes.

\section{Acknowledgement}

This work was supported in part by Volkswagen Stiftung (I/63733). We are indebted to G.G. Gross for giving us a generous gift of tannase. We gratefully acknowledge W.R. DeMott and L. Härter for reviewing the manuscript and Nancy Zehrbach for linguistic improvement.

\section{References}

Aliotta, G., Molinario, A., Monaco, P., Pinto, G., and Previtera, L., 1992: Three biologically active phenylpropanoid glucosides from Myriophyllum verticillatum. Phytochemistry 31:109-111.

Box, J.D., 1983: Investigation of the Folin-Ciocalteu phenol reagent for the determination of polyphenolic substances in natural waters. Water Res. 17:511-525.

Grace, J.B., and Wetzel, R.G., 1978: The production biology of Eurasian watermilfoil (Myriophyllum spicatum L.): a review. J. Aquat. Plant Manage. 16:1-11

Gross, E.M., Wolk, C.P., and Jüttner, F., 1991. Fischerellin, a new allelochemical from the freshwater cyanobacterium Fischerella muscicola. J. Phycol. 27:686-692.

Haslam, E., 1989: Plant Polyphenols. 230 pp., Cambridge University Press, New York, NY 10022, USA.

Janson, E., 1918: Über die Inhaltskörper der Myriophyllum-Trichome. Flora (Germ.) 110:265ff.

Kane, M.E., and Gilman, E.F., 1991: In vitro propagation and bioassay systems for evaluating growth regulator effects on Myriophyllum species. J. Aquat. Plant Manage. 29:29-32.

Nalewajko, C., and Godmaire, H., 1993: Extracellular products of Myriophyllum spicatum L. as a function of growth phase and diel cycle. Arch. Hydrobiol. 127:345-356.

Planas, D., Sarhan, F., Dube, L., Godmaire, H., and Cadieux, C., 1982: Ecological significance of phenolic compounds of Myriophyllum spicatum. Verh. Internat. Verein. Limnol. 21:1492-1496.

Saito, K., Matsumoto, M., Sekine, T., and Murakoshi, I., 1989: Inhibitory substances from Myriophyllum brasiliense on growth of blue-green algae. J. Nat. Prod. 52: 1221-1226.

Scheffer, M., Hosper, S.H., Meijer, M.-L., Moss, B., and Jeppesen, E., 1993: Altemative equilibria in shallow lakes. TREE 8:275-279

Steemann Nielsen, E., 1952: The use of radio-active carbon $\left(\mathrm{C}^{14}\right)$ for measuring organic production in the sea. J. Cons. Int. Expl. de la Mer 18:117-140.

Wetzel, R.G., 1992: Gradient-dominated ecosystems - sources and regulatory functions of dissolved organic matter in freshwater ecosystems. Hydrobiologia 229:181-198.

Wium-Andersen, S., 1987: Allelopathy among aquatic plants. Arch. Hydrobiol. Beih. Ergebn. Limnol.27:167-172. 

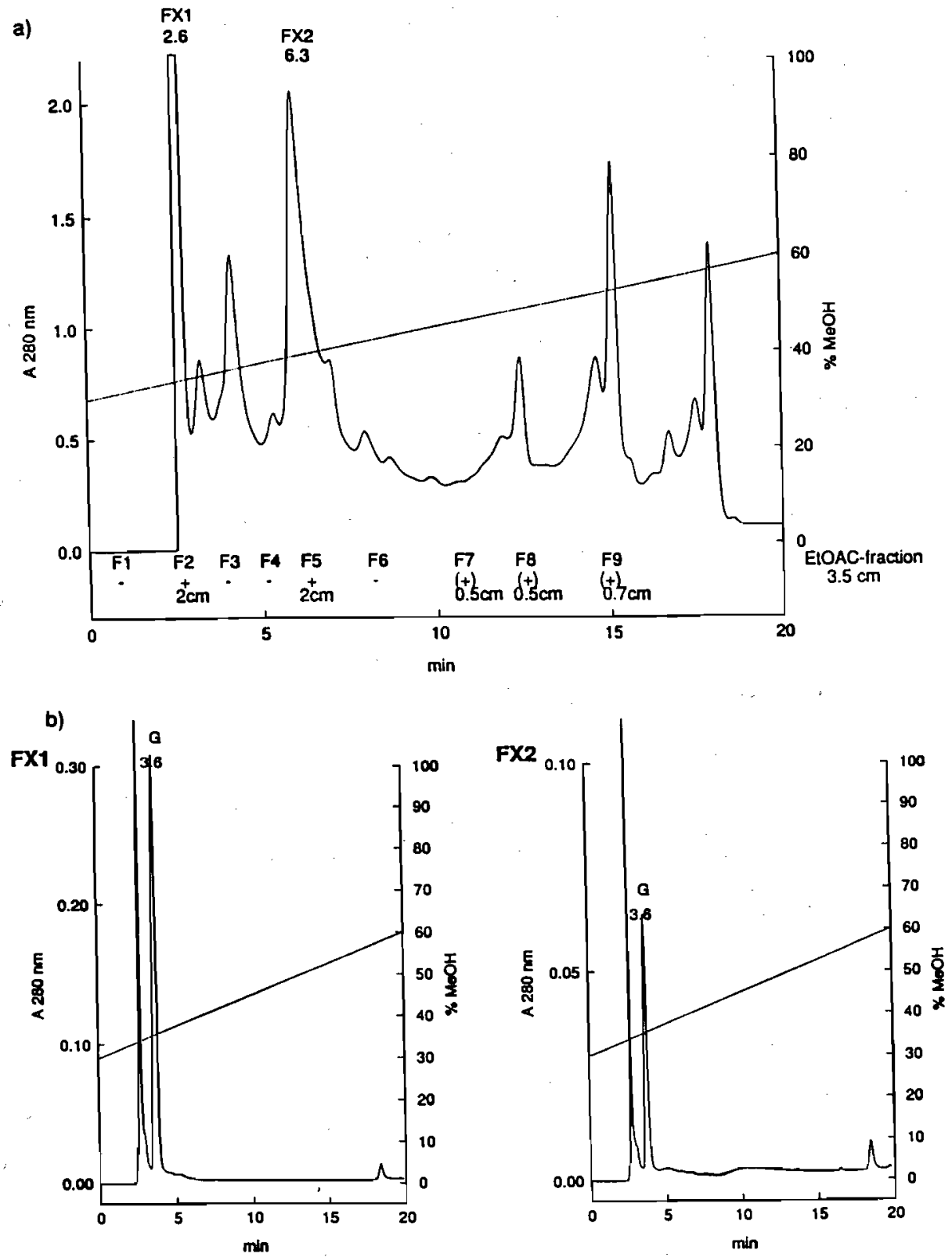

Fig. 1:

a) RP-HPLC separation of the EtOAc fraction from $M$. spicalum. Numbers above the $x$-axis indicate fractions in the ADA. + inhibition ( $\phi$ in $\mathrm{cm}$ ), - no inhibition.

The main inhibitors, fractionated in $F 2$ and $F 5$ are signed with $F X 1$ and $F X 2$, respectively.

b) Enzymatic hydrolysis of FX1 (left) and FX2 (right) with tannase.

RP-HPLC separation of the cleavage products. Detection of gallic acid (G) at Rt $3.6 \mathrm{~min}$. 

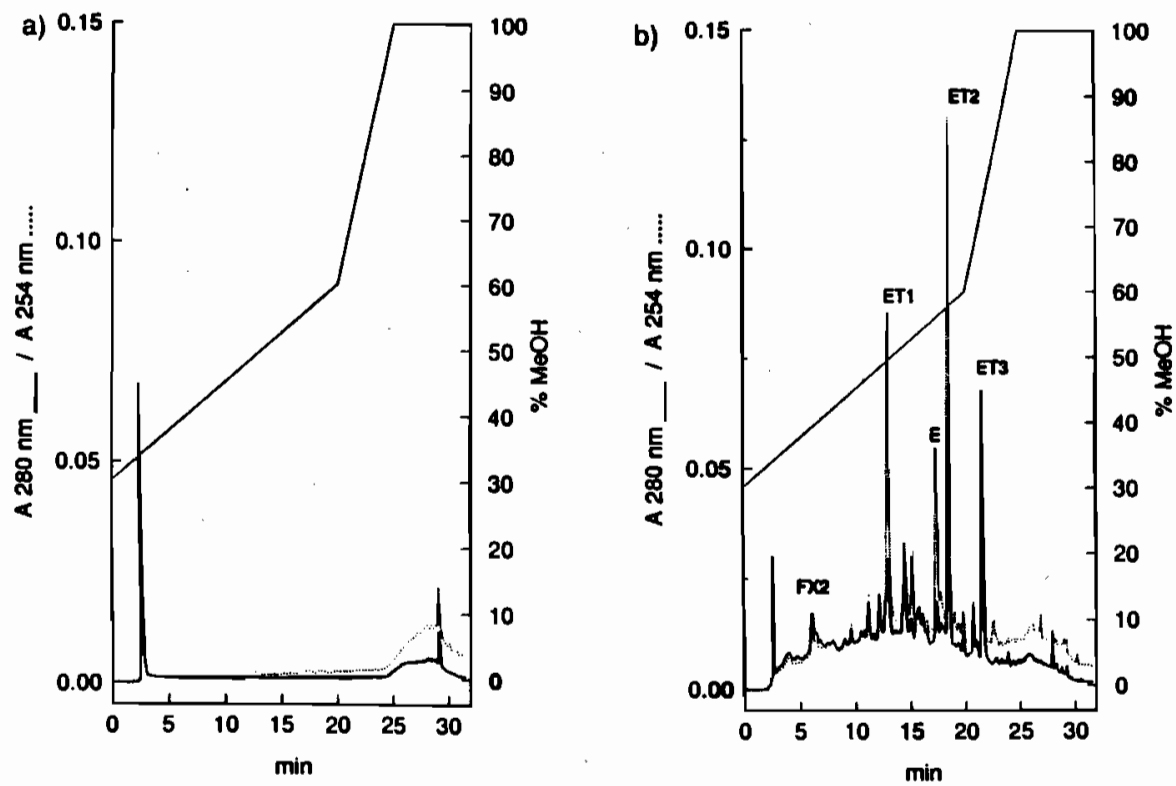

Fig 2:

RP-HPLC separation of SPE-enriched culture medium (a) and culture water from M. spicatum (b). FX2: algicidal galloyl ester, E: ellagic acid, ET1 - ET3: different ellagic acid derivatives.
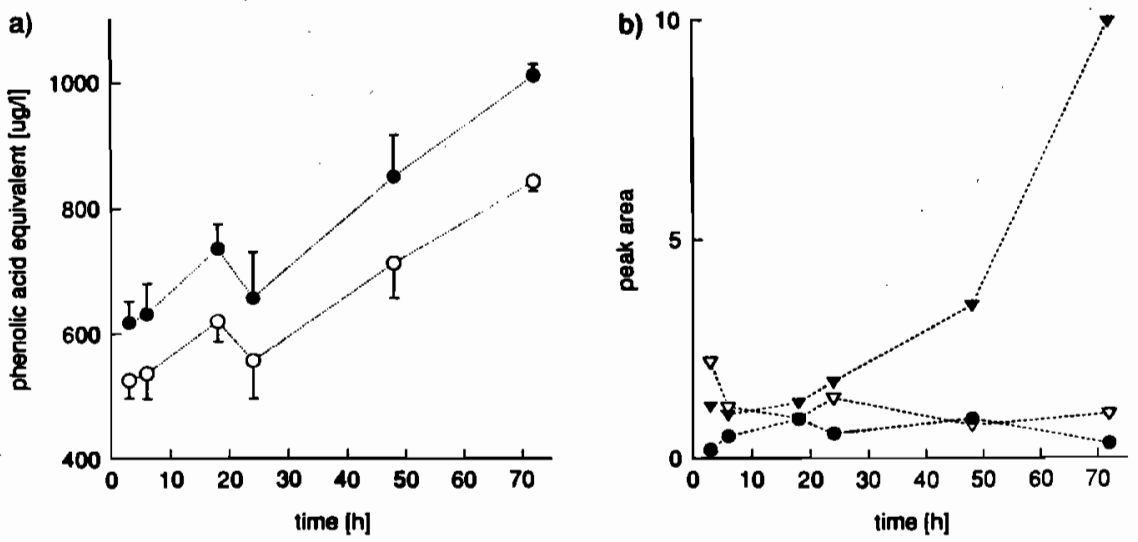

Fig 3:

Accumulation of phenolic compounds in cuiture water of $M$. spicatum

a) Folin assay for total released phenolics. The measured absorbance at $720 \mathrm{~nm}$ was converted to gallic (filled dots) and tannic (hollow dots) acid equivalents. Standard deviation was calculated from triplicates.

b) RP-HPLC separtation of SPE enriched culture water. Time-course of selected polyphenols.

Quanification was done by peak area integration. Data are shown for one of the algicidal galloyl esters (FX2, absorbance measured at $280 \mathrm{~nm}$, filled dots) and two ellagic acid derivatives (absorbance measured at $254 \mathrm{~nm}$, Rt $18.5 \mathrm{~min}$ hollow triangles, Rt $21.5 \mathrm{~min}$ filled triangles). 